\title{
LEGGERE IBN 'ARABĪ IN ITALIANO. QUANDO IL SUFISMO DIVENTA AFFARE DI STATO
}

\section{Claudio Marzullo}

Università degli Studi di Napoli "L'Orientale"

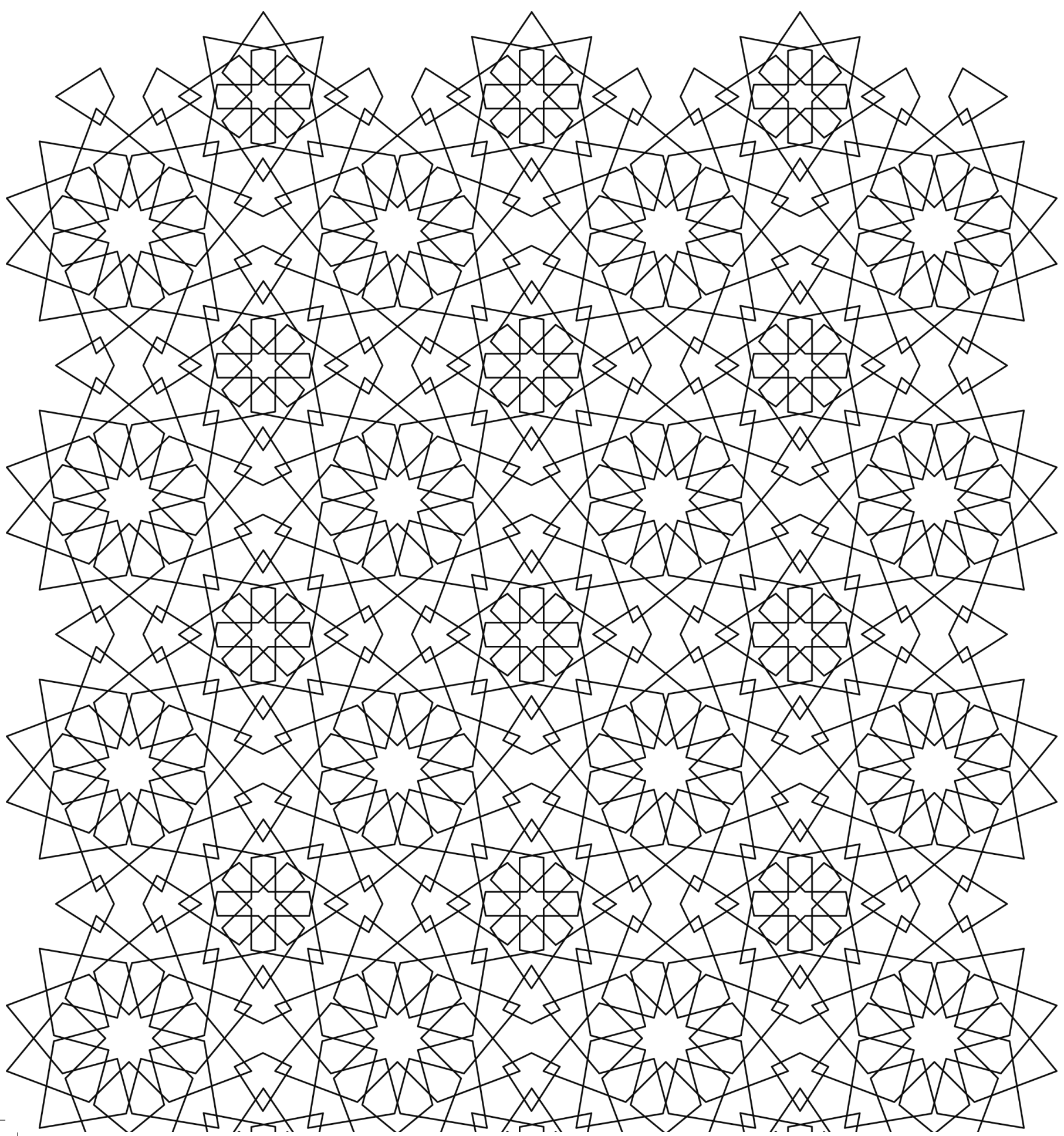


Una serie di articoli in italiano dedicati a Ibn 'Arabī (1165-1240) e al Sufismo apparve, nella prima decade del secolo scorso, sul periodico italo-arabo "Il Convito-Al-Nādī”, fondato dal Dottor Enrico Insabato (1878-1963) al Cairo nel 1904. Tali pagine, al netto d'ogni giudizio sul valore culturale, scientifico o spirituale, sono uno curiosissimo caso, forse unico, in cui gli scritti del Sommo Maestro assumono un così gran rilievo nella politica estera di un paese, al punto da divenire "affare di Stato".

Ma se quest'affermazione ha dello stupefacente, a fortiori lo stupore accrescerà se diciamo che lo Stato è l'Italia di Giolitti ${ }^{1}$ - dove Ibn 'Arabī era allora pressoché uno sconosciuto - e che la posta in gioco, sul piano dei rapporti esteri, è la penetrazione coloniale nel Nord Africa, e in generale nel mondo musulmano, ai danni di Francia e, soprattutto, Gran Bretagna. Tale penetrazione, si sarebbe avvalsa, in primo luogo, della ricerca di consenso presso le élites religiose tradizionali dell'Impero Ottomano: l'Italia, unica potenza in Europa a considerare la civiltà islamica, nel dialogo intellettuale e morale, su un piede di parità; una nazione che non vuole "macchiarsi le mani di sangue musulmano" per imporsi in Oriente. Quale che sia il giudizio sulla bontà d'ideali e sincerità d'intenti, e cioè se, in questo momento storico, l'Italia intendesse le relazioni con l'Oriente anche in maniera differente dall'ordinaria politica degl'imperi coloniali - argomento interessantissimo ma che va ben al di là del nostro intervento - fatto è che questa fase si svolse nel primo decennio del ventesimo secolo, e cioè prima della guerra di Libia (1911).

E se a queste considerazioni aggiungiamo che a scrivere tali articoli su Ibn 'Arabī non fu un italiano, né un madrelingua italiano, bensì il pittore svedese John Gustav Agelii (1869-1917), ci si chiederà allora come sia stato possibile tutto questo e perché. Per rispondere a queste domande è necessario fare un passo indietro ed esaminare la figura di Insabato il quale, come abbiamo accennato, fondò "il Convito" al Cairo nel 1904; parleremo poi della rivista in generale e passeremo infine a parlare degli articoli su Ibn 'Arabī e del loro autore.

Figlio di un ferroviere, Enrico Insabato frequentò dapprima la Facoltà di Lettere di Bologna, entrando in contatto con circoli anarchici nel 1898; nel 1900 era a Parigi, per la "École Supérieure de Medécine Coloniale”. Forse fu in questo soggiorno parigino che conobbe il pittore, anarchico anch'egli, Agelii, di cui parleremo più avanti. Ottenne il diploma prima di dicembre 1902, poiché in quella data si trasferì al Cairo. La motivazione ufficiale dell'arrivo in Egitto fu la partecipazione al primo Congresso medico internazionale tenutosi al Cairo il 16 di quello stesso mese ${ }^{2}$. Non un semplice

1 Ci riferiamo, in particolar modo, al secondo e terzo mandato alla Presidenza del Consiglio dei Ministri (1903$5 ; 1906-9)$.

2 Le nostre fonti principali su Insabato sono: C. Gotti Porcinari, Rapporti Italo-Arabi (1902-1930) dai documenti di Enrico Insabato, E.S.P., Roma, 1965, pp. 17-23; Anna Baldinetti, Orientalismo e Colonialismo. La ricerca del consenso in Egitto per l'impresa in Libia, Istituto per l'Oriente "C. A. Nallino", Roma, 1997, pp. 33-70. Entrambi gli autori, sebbene con maggior rigore storico e scientifico nel secondo caso, citano documenti d'archivio, tra cui l'Archivio Centrale di Stato e l'Archivio storico-diplomatico del Ministero degli Affari Esteri di Roma. Per un'interpretazione più recente della politica italiana nel Vicino Oriente e la funzione de "Il Convito": Meir Hatina, 
medico e simpatizzante anarchico, Insabato fu soprattutto agente del Governo, in costante e diretto contatto col Primo Ministro Giolitti, durante il secondo e terzo mandato di questi ${ }^{3}$. Si può affermare con sicurezza che il progetto di lavorare come agente del Governo al Cairo fosse già stato ideato o pianificato prima dell'arrivo in Egitto poiché, lo stesso Insabato, in una lettera diretta al presidente del Consiglio, non datata, afferma:

«Sin dal 1901 ebbi l'onore di presentare a S. E. Giolitti, allora presidente del Consiglio, il progetto di stabilirmi in Cairo d'Egitto, di iniziarvi la pubblicazione di un giornale e di far sì che la redazione di questo periodico divenisse centro di tutte quelle informazioni d'indole politica e di quelle iniziative che non avessero potuto, come ben si comprende, essere assunte dalla R. Legazione di Cairo [...] La scelta, da parte mia, del Cairo era stata determinata dall'essere questa città il centro vitale, direi quasi il cervello di tutto il mondo musulmano» ${ }^{4}$.

Giunto al Cairo, Insabato fondò dapprima la rivista Il Commercio italiano, nel 1903, la quale ebbe durata breve, poiché già l'anno successivo terminò la pubblicazione ${ }^{5}$. Essere direttore di un periodico in lingua italiana in Egitto agli inizi del XX secolo non era certo un fatto eccezionale né particolarmente originale: gli ultimi anni dell'Ottocento, infatti, testimoniarono un fiorire straordinario di pubblicazioni in italiano - molte delle quali a carattere effimero e temporaneo - dal taglio più disparato - politico, economico, artistico, sportivo o umoristico - a cadenza quotidiana, settimanale, quindicinale o mensile - ad Alessandria, il Cairo e Port Said ${ }^{6}$. Ciò non deve stupire essendo quella italiana, dopo quella greca, la comunità di espatriati più numerosa in Egitto.

Originalissima e forse unica nel suo genere, invece, fu la creazione de "Il Convito-Al-Nādī" nel 1904. Il pubblico della stampa occidentale in Egitto era, come è ovvio, la comunità di espatriati alla quale era diretta la singola testata, e raramente questi limiti venivano superati. Poteva circolare liberamente in tutto il Vicino Oriente perché non attaccava le autorità ottomane.

Where East Meets West: Sufism, Cultural Rapprochement, and Politics, International Journal of Middle East Studies, Vol. 39, n. 3 (Agosto 2007), pp. 389-409. Tuttavia, per quel che riguarda le fonti storiche, lo studio si basa sui fondamentali lavori sopra citati di Gotti Porcinari e Baldinetti.

3 Che Insabato fosse in contatto con Giolitti dall'Egitto almeno fin dal 1903 è un fatto dimostrato, con riferimento preciso a dati dell'Archivio storico-diplomatico del Ministero degli Affari Esteri di Roma, da Anna Baldinetti (Orientalismo e Colonialismo: p. 35).

4 Gotti Porcinari, Rapporti Italo-Arabi, p. 17.

5 Alessandra Marchi, La presse d'expression italienne en Égypte. De 1845 à 1950, Rivista di Storia dell'Istituto dell'Europa Mediterranea (RiMe), no. 5, dicembre 2010, 107-8. L'autrice afferma che la testata fu fondata nel 1902. Tuttavia, stando ai dati biografici in Gotti Porcinari e Baldinetti, Insabato sarebbe arrivato in Egitto a dicembre 1902, dunque il primo numero sarebbe uscito, verosimilmente, nel 1903. In realtà, quasi nulla si conosce di questa rivista, oltre al nome.

6 Marchi, La presse: pp. 102-6. 
«Pensai allora di ampliare la mia sfera di azione e, presi opportuni accordi, mutai il periodico "Il Commercio italiano" in un periodico bilingue, italiano-arabo, "Il Convito" (maggio 1904) [...] Il mio periodico non attaccando il governo del Sultano circolò liberamente in tutto l'Impero turco».

"Il Convito", essendo scritto in italiano e arabo e, per un periodo limitato, anche in turco, si rivolgeva non soltanto agli italiani che erano in rapporto costante con l'Egitto e l'Oriente islamico, ma in primo luogo alle élites intellettuali musulmane tradizionali, egiziane e non, le quali erano in modo diretto o meno legate al Sufismo; non agli egiziani occidentalizzati, i quali, al contrario, erano fortemente influenzati da idee nazionalistiche e moderne e che spesso profittavano, per lo stile di vita "all'occidentale" al quale erano adusi, dello statu quo, il protettorato britannico in Egitto; parimenti, la testata era contraria in modo chiaro e deciso al modernismo/riformismo islamico della Salafyya - cui corifeo era Raš̄i Riḍā (1865 - 1935). Tale movimento, agli occhi sia di Insabato che di Agelii, non rappresentava lo spirito genuino della tradizione islamica, bensì il semplice reagire alla decadenza del mondo islamico rivivificandone i dogmi in una forma mentis occidentale moderna; la qual cosa avrebbe così ridotto una tradizione spirituale e religiosa, come quella islamica, i cui principi si traducono anche nell'ordine sociale e nelle istituzioni - e la cui applicazione è però da moderare in modo saggio e opportuno a seconda del contesto storico e culturale - in un'ideologia politica totalitaria: il precetto de "l'ordinare il bene e proibire il male" in uno Stato di polizia. La rigidità mentale e la ristrettezza di vedute di questo movimento avrebbe reso i suoi adepti particolarmente vulnerabili a ogni retorica manipolatrice delle potenze coloniali. L'unica forma di colonialismo accettabile, secondo quanto affermato dal periodico, avrebbe potuto essere soltanto la presenza pacifica a scopo commerciale. ${ }^{7}$

La creazione de "Il Convito" rientrava in un progetto lucido e articolato di penetrazione italiana nel Vicino Oriente, sviluppato da Insabato, e che, almeno in una prima fase, avrebbe dovuto essere di carattere pacifico attraverso la ricerca di consenso nelle società islamiche tradizionali per la politica estera italiana. Tale consenso, lo si sarebbe ottenuto attraverso atteggiamenti concreti filo-islamici dell'Italia. Tali linee guida sono state sintetizzate da Anna Baldinetti ${ }^{8}$. Non ci soffermeremo più di tanto su questo, ma diremo soltanto che il piano avrebbe previsto: l'istituzione al Cairo di una scuola per l'insegnamento dell'italiano e diffusione dell'influenza italiana; la fondazione di un giornale italoarabo; ottenere l'appoggio di confraternite sufi, in particolar modo la Senussia in Tripolitania, la quale controllava sia l'opinione pubblica che le piste carovaniere in quell'area; finanziare pubblicazioni in italiano e arabo; non contrastare l'applicazione delle norme sciaraitiche nelle colonie italiane; la costruzione a Roma di una moschea da parte del governo italiano; chiedere all'Impero Ottomano sia l'impiego d'italiani nell'amministrazione turca che permettere ai sudditi dell'Impero la frequen-

7 Si veda l'analisi delle posizioni teoriche e dell'orientamento politico della rivista fatta da Angelo Scarabel, Una rivista italo-araba d'inizio secolo: $A \mathcal{N}-\mathcal{N A} D \bar{I}$ (IL CONVITO), Oriente Moderno, Anno 58, Nr. $1 / 3$ (Gennaio-Marzo 1978), pp. 51-67.

8 Baldinetti, Orientalismo e Colonialismo, pp. 35-70. 
tazione di scuole italiane; imprese commerciali e un servizio di trasporto per il viaggio dei pellegrini alla Mecca. Quello che in concreto fu realizzato sia dal Governo italiano che dalla libera iniziativa di Insabato fu, oltre alla creazione de "il Convito", la costruzione di una moschea al Cairo dedicata alla memoria di Re Umberto I nel 1906-79'; dal 1908 a 1912, fu sovvenzionato l'insegnamento dell'italiano in una scuola cairota; la costruzione, nel 1912, dopo l'invasione della Libia, di un alloggio per gli studenti (riwāa) somali ed eritrei e l'elargizione di sussidi per gli studenti tripolini della prestigiosa università religiosa di al-Azhar, sempre al Cairo.

Ritornando a "il Convito", esso raggiunse un bacino di circa 5.000 lettori, e fu distribuito in tutto il Vicino Oriente, Egitto, Tripolitania e Cirenaica in particolare ${ }^{10}$. La pubblicazione tuttavia continuò, con varie interruzioni e riprese, soltanto fino al $1910^{11}$, a causa del ritorno in Italia di Insabato nel maggio di quell'anno; ma non è chiaro se vi fece ritorno spontaneamente ho fu obbligato a farlo. Insabato era stato già stato richiamato dal Governo italiano nel 1907-9, poiché, a causa della pubblicazione di alcuni articoli su "Il Convito" in cui apertamente criticava Lord Cromer, le autorità britanniche ne avevano chiesto l'allontanamento dall'Egitto ${ }^{12}$. Ritornò dunque in Egitto dopo due anni, non più come agente del Governo, ma come agente del Ministero dell'Interno e alle dipendenze dell'agente diplomatico. Insabato però agì di sua iniziativa - in particolar modo stringere relazioni con i Senussi in Tripolitania in vista dell'invasione italiana della Libia - la qual cosa avrebbe ulteriormente infastidito le autorità anglo-egiziane ${ }^{13}$.

Gli avvenimenti successivi della biografia di Insabato sono poco rilevanti per il nostro tema; diremo soltanto che Insabato ritornò al Cairo nel 1911, per informare il Governo italiano dell’impatto della guerra di Libia sull'opinione pubblica egiziana; tuttavia, egli continuò ad agire di sua iniziativa, soprattutto per le trattative dell'Italia con i capi senussi, spesso sovrapponendosi all'operato dell'agenzia diplomatica. Fu inviato a Istanbul nel 1914 dal Ministero degli Esteri ${ }^{14}$.

Ibn 'Arabī su "Il Convito"

In questo contesto, la pubblicazione di articoli sul Sufismo assunse una valenza politica molto chiara. Nel primo numero del periodico, il 22 maggio 1904, Insabato scriveva:

9 Ritorneremo più avanti su questo fatto.

10 Gotti Porcinari, Rapporti Italo-Arabi, p. 17, il quale riporta le parole dello stesso Insabato.

11 Secondo quanto riportato da Gotti Porcinari (Rapporti Italo-Arabi, pp. 22-23), sembrerebbe che nel 1912 la rivista fosse ancora pubblicata, dopo un'interruzione nel 1911; per Baldinetti (Orientalismo e Colonialismo, p. 38), l'esperienza de "Il Convito" si concluse nel 1910. Tuttavia, Baldinetti, a pagina 65 dello stesso studio, accenna a un «supplemento al numero del gennaio-febbraio 1912 della rivista Il Convito».

12 In particolare, un articolo in arabo (n. 2, giugno 1907, pp. 40-42); v. Baldinetti, Orientalismo e Colonialismo, pp. 57-58.

13 Baldinetti, Orientalismo e Colonialismo, pp. 54-61.

14 Baldinetti, Orientalismo e Colonialismo, pp. 68-70. 
«Le due grandi civiltà: l'Araba e l'Europea, possono avere dei punti di partenza diversi; ma hanno lo stesso scopo, poiché esse sono parallele o meglio convergenti. [...] Il vero nemico della fede religiosa è lo scetticismo; d'altra parte il vero nemico di una civiltà antica quale la latina è l'invidia e l'ambizione personale. Questi due nemici sono solo in apparenza diversi; hanno un solo ed unico rimedio: l'idealismo, la cui forma orientale si chiama: "el Tasawuf". Lo spiritualismo è la sola cosa che possa impedire la decomposizione di una società o di una religione, cosi come l'anima e la vita impediscono la decomposizione del corpo. [...] Noi daremo dunque, in questo giornale largo spazio alle manifestazioni del "sufismo" [...] Noi vogliamo ugualmente combattere la maldestra e ridicola imitazione dell'Europa, poiché la troviamo nocevole tanto all'Oriente che all'Occidente [...]. Per essere progressisti in Oriente bisogna cominciare ad essere intelligentemente reazionari [...] Perché il futuro sia grande e realizzabile è necessario che esso abbia le sue radici nella coscienza del passato» ${ }^{15}$.

A curare gli articoli sul Sufismo e Ibn 'Arabī fu John Gustav Agelii (1869-1917) - chiamato "Ivan Aguéli” e, in seguito alla conversione all'Islam, “Abd al-Hādī” - pittore svedese di rilievo ${ }^{16}$. Da giovane egli si trasferì a Parigi dove, nell'ultima decade dell'Ottocento, frequentò ambienti culturali, artistici e politici più disparati: caffè letterari, atelier, circoli di occultisti, teosofisti ${ }^{17}$ e anarchici. Fu probabilmente in ambienti anarchici che conobbe Enrico Insabato. Allo stesso tempo s'immerse nello studio di lingue e culture orientali, tra cui: arabo, ebraico, ge'ez e malese. Questo interesse sembra sia stato alimentato da due viaggi che fece in questi anni (in Egitto, 1894-5; a Ceylon, 1899). Punto di partenza fu il misticismo di Swedenborg, ma anche l'occultismo e il teosofismo ${ }^{18}$. Il suo interesse non era alimentato da desiderio d'erudizione, né da velleità accademiche, né da un fatuo esotismo, bensì da un'intensa ricerca spirituale. In seguito a tali studi e ricerche abbracciò l'Islam e, in particolar modo, la sua attenzione fu rivolta all'esoterismo islamico.

Si trasferì quindi in Egitto nel 1902. Al Cairo fu iniziato al Sufismo dallo šayh 'Abd al-Raḥmān 'Illī̌s ('Tlīš, 'Tlayš ovvero 'Ulayš) al-Kabīr (circa 1845 - 1922), un influente maestro della confraternita

15 Gotti Porcinari, Rapporti italo-arabi, p. 19.

16 Abbiamo ricavato le informazioni sulla vita di Agelii principalmente da Scarabel (Una rivista italo-araba) e dall'introduzione a 'Abdul-Hâdî (John Gustav Agelii, dit Ivan Aguéli), Écrits pour La Gnose comprenant la traduction de l'arabe du Traité de l'Unité, Archè, Milano, 1988. Non abbiamo potuto consultare le due biografie in svedese: Van Hausen, Minnen fran samvaron med Gustav Agelii i Paris och Cairo 1894-1895, in Ord och Bild, Årg 35, $1926, \mathrm{n}$. 608; Axel Gauffin, Ivan Aguéli. Människan, mystikern, målaren, Stockholm, 1940-41.

17 Fu legato in qualche modo alla Società Teosofica, o comunque ebbe simpatie teosofiste, almeno fino al 1907, poiché in questa data scrisse un necrologio dal tono fortemente encomiastico per il Colonnello Olcott.

18 Queste considerazioni sono basate sui libri che Agelii portò con sé nel suo primo viaggio in Egitto (1894-5); v. 'Abdul-Hâdî, Écrits pour La Gnose, introduzione, pp. X-XI. 
Šādilita, professore di diritto Malikita all'università di al-Azhar al Cairo. Lo šayh, molto influente anche sul piano politico, era stato coinvolto assieme a suo padre nella rivolta di 'Urabī Paša nel 1882 in Egitto ed aveva vissuto alcuni anni in esilio in Siria e a Istanbul. A breve parleremo di questa persona.

Agelii fu il principale collaboratore di Insabato per la rivista "Il Convito"; dopo l'esperienza del periodico italo-arabo, e avendo fatto ritorno in Europa, collaborò con la redazione della rivista francese $L a$ Gnose, scrivendo articoli sull'esoterismo islamico tra il 1910 e il 1911. Il metafisico e studioso di simbolismo francese René Guénon (1886-1951), il quale pure scriveva per la Gnose, fu molto probabilmente influenzato dalla personalità e dalle concezioni di Agelii. Non ci dilungheremo oltre sulla biografia di Agelii; diremo soltanto che gl'ultimi anni della sua vita furono segnati da gravi problemi finanziari e mancanza di mezzi di sussistenza; morì a Barcellona investito da un treno nel 1917.

Per quel che riguarda "il Convito", Agelii scrisse articoli sul Sufismo sia nella sezione italiana che araba. Nella parte italiana curò una rubrica chiamata "El Akbariya", con riferimento alla corrente spirituale dello Šayh al-Akbar, "il Sommo Maestro" del Sufismo Muhȳi l-Dīn Ibn al-'Arabī. Nelle intenzioni di šayh 'Illī̌s e di Agelii, la "El Akbariya" avrebbe dovuto essere anche un'organizzazione non si sa fino a che punto assimilabile a una confraternita sufi, a una loggia massonica ovvero a una élite puramente intellettuale - il cui obbiettivo avrebbe dovuto essere la diffusione del Sufismo - ma, soprattutto, una rinascita spirituale - in Occidente.

Quel che si sa con certezza è che šayh 'Illī̌s collaborò coscientemente con Enrico Insabato e il Governo italiano, almeno fino alla guerra di Libia nel $1911^{19}$, cercando forse nell'alleanza con l'Italia di limitare l'ingerenza britannica nei paesi del Vicino Oriente. In questo contesto rientrava la costruzione della moschea dedicata alla memoria di Re Umberto I nei pressi di al-Azhar al Cairo, voluta da šayh 'Illī̌s e appoggiata dal Governo italiano. La questione della liceità nel dedicare una moschea a un non-musulmano provocò forti critiche; in particolare Raš̄ì Riḍā definì il fatto un'innovazione bizzarra (e deprecabile) senza precedenti; šayh 'Illī̌s veniva accusato di ricevere denaro dall'Italia e l'iniziativa veniva presentata come un'operazione funzionale alla strategia politica del Governo italiano in Egitto $^{20}$. Riportiamo dei passi dell'articolo apparso su "Il Convito", 1907, n. 3-4, firmato da Agelii, dal titolo La moschea 'Umberto' (in faccia a 'El Azhar'), a proposito della vicenda:

«Uno degli uomini più celebri dell'Islam, figlio del restauratore del rito Malekita e lui stesso profondo sapiente ${ }^{21}[\ldots]$ un'autorità incontestabile dell'Islam essoterico ed esoterico, giuridico e politico, ha fatto costruire a sue spese, su un terreno adiacente alla casa da lui abitata a El Azhar stesso, una moschea di cui ha fatto dono all'Italia e che ha dedicato al defunto re

19 Tuttavia, ci sarebbe stato già nel 1909 un avvicinamento dello šayh alla Francia (v. Écrits pour la Gnose, p. XIV, nota 6). 20 Articoli di Rašìd Riḍā su al-Manār, 10, luglio 1907, pp. 398-400; 10, ottobre 1907, pp. 637-38. Baldinetti, Orientalismo e Colonialismo, pp. 43-44.

$21 \mathrm{Si}$ riferisce a šayh 'Illī̌s. 
Umberto. È la prima volta, dopo molti secoli, che un simile fenomeno si produce: e noi ne dobbiamo ai lettori nostri la spiegazione. Poi che il fatto è non solo della più grande importanza per la civiltà in generale ed il progresso italiano in Oriente in particolare, ma ci permette esso di vedere l'Islam sotto la sua vera luce: come la Religione ch'è al di sopra delle religioni e come fede veramente universale non occupantesi delle ostilità di lingue, di popoli e di razze, ma riunente la umanità tutta in un solo sistema completo e generale di organizzazione spirituale e di ordine ieratico e mistico».

La redazione de "Il Convito", come ovvio, difese lo šayh sia trovando appoggio nelle fonti islamiche che criticando l'impostazione "protestantesca" e modernista di Rašìd Riḍā, la cui rigidità avrebbe portato al fanatismo. La vicenda della moschea Umberto però rese impopolare šayh 'Illī̌s.

Veniamo ora agli articoli su Ibn 'Arabī. Nella rubrica "El Akbariya”, Agelii tradusse alcuni estratti delle Futūhāt al-makkiyya, "Le Rivelazioni mecchine"; vi erano poi articoli in cui Agelii presentava la dottrina di Ibn 'Arabī raggruppata in grandi temi. Ecco le dichiarazioni programmatiche al termine del primo numero della rubrica:

«Noi parleremo delle rivelazioni "del più grande degli sceikhs" in una serie di capitoli, ciascuno dei quali tratterà dell'uno o dell'altro mistero: i misteri Dominicali, profetici, biblici, angelici e celesti; quelli del culto, della glorificazione, dell'amore, della fede, della carità; quelli dell'uomo e del suo "io," della creazione, del tempo, dell'eternità, del fato, della vita e della morte; quelli delle prove, delle tentazioni, dei peccati, del perdono e della vita nuova. All'ultimo noi parleremo della vita dello Sceikh, dei partigiani e detrattori ch'egli ebbe e diremo delle sue analogie ed armonie celesti, cioè del suo sistema d'occultismo»» ${ }^{22}$.

La scelta delle "Rivelazioni”, che è senza dubbio l'opera massima di Ibn 'Arabī, a discapito di altre opere, sia pur famose e notevoli come Fusūs al-hikam, "I Castoni della saggezza", venne motivata da Agelii in questo modo:

«Questa opera rimane sempre non solo il più nel monumento della letteratura mistica islamo-araba, ma ben anche il libro nel quale lo Sceikh rivela la propria personalità, così profonda, lucida e possente. Essa, almeno secondo la nostra modesta opinione, è più improntata dell'anima dello Sceikh, che i suoi «Divani» (raccolta di poesie) ed i suoi Aforismi sui profeti, intitolati. Fusûs el Hikami»»23.

22 Il Convito, 1907, n. 2, p. 55.

23 Il Convito, 1907, n. 3-4, p. 90. 
Tuttavia, dal secondo numero della rubrica, Agelii modificò un poco la modalità di esposizione, venendo incontro alle difficoltà dei lettori italofoni, per i quali doveva essere il primo incontro con Ibn 'Arabī, e forse con il Sufismo tutto:

Molti lettori però mi hanno fatto intendere che, data la novità del soggetto, io farei meglio esponendo le teorie e la lor influenza logica e morale, innanzi all'analisi dei sentimenti. Io aveva creduto il contrario; m'ero detto ch'era più facile di comunicare per mezzo di un sentimento che di un pensiero e che una emotività è la chiave di un sistema filosofico o religioso. [...] Innanzi dunque di toccare al programma annunciato alla fine dell'articolo «El Akbariya» del numero precedente, io farò alcuni articoli nei quali esporrò, con un ordine arbitrario in apparenza, le teorie generali del misticismo arabo, così come Mohhyi ed Din le ha formulate; e ciò allo scopo di giungere poco per volta all'esposizione della di lui personalità» ${ }^{24}$.

Per nostra somma sfortuna i numeri del periodico non sono di facile reperimento, almeno in Italia: la chiusura al pubblico della biblioteca dell'IsIAO (Istituto italiano per l'Africa e l'Oriente) a Roma, la quale è l'unico luogo dove c'è -o dovrebbe esserci - l'intera raccolta in Italia, rende particolarmente onerosa la ricerca e l'analisi sistematica e complessiva di questi primissimi e originalissimi studi su Ibn 'Arabī in italiano. Alla luce di questi problemi di accesso alle fonti, non possiamo avere un'idea precisa dell'insieme dei concetti dottrinali affrontati.

È tuttavia sufficiente aver letto qualche articolo per farsene un'idea piuttosto nitida del taglio; non è certo quello dell'Orientalismo classico, né pura ammirazione per l'esotico. In quanto convertito all'Islam, e ricollegato al sufismo, Agelii intendeva la dottrina akbariana come la più pura "espressione della verità" in accordo alla saggezza islamica, non un comune sistema filosofico. Sebbene fosse formatosi anche in ambienti teosofisti e occultisti della Parigi di fine Ottocento, per i quali - ci scusiamo per l'eccessiva semplificazione - sarebbe stato possibile accedere, con positivistica sicurezza, a "discipline occulte" e a una "saggezza primordiale" anche con mezzi e approcci moderni, Agelii invece intendeva rendere il lettore occidentale consapevole delle enormi differenze tra la saggezza delle civiltà orientali e l'esoterismo moderno.

La terminologia di Agelii era spesso fumosa, incerta, bizzarra, quasi nä̈f; non ci sembra che vi fosse rigore e né una piena consapevolezza nella traduzione del vocabolario tecnico del Sufismo in italiano; ciò fu dovuto senza dubbio in parte al fatto che Agelii non fosse madrelingua italiano, ma soprattutto al fatto che la sua fu un'opera davvero pioneristica. Il lessico utilizzato per descrivere il Sufismo, e in generale le dottrine orientali, si sarebbe poi, nel corso del Novecento, specializzato nella direzione 
dell'Orientalismo ufficiale ${ }^{25}$ che in quella degli studi non-accademici, fornita da autori non-accademici, in cui un ruolo rilevantissimo ha giocato, in primis, la lettura degli scritti di René Guénon e, in un secondo momento, anche di autori come Titus Burckhardt, Michel Vâlsan, e altri ancora. Per dare un esempio, prendiamo una nota a piè pagina riferitesi a un passo $^{26}$ in cui Agelii affrontò la questione della conoscenza di Dio da parte dell'uomo:

«La subcoscienza, in sufismo, è una delle forme della coscienza, talora una delle sue forme più elevate».

La "subcoscienza" di cui parlava certo non era una categoria psicoanalitica, non era "sotto" lo stato cosciente, bensì "sopra": l’intuizione è inesprimibile non perché il soggetto non ne sia capace, ma perché l'oggetto stesso di tale coscienza non è manifestabile. Una terminologia impropria può dare adito a spiacevoli confusioni. In altri casi, tuttavia, Agelii fu ben consapevole nell'uso di termini e categorie filosofiche occidentali; ad esempio, con grande anticipo rispetto a molti orientalisti della prima metà del Novecento, distinse senza indugi la concezione di "panteismo" dalla dottrina di Ibn 'Arabī:

«La parola "panteismo" è un termine assai vago per indicare una cosa assai vaga anch'essa. È perciò che i musulmani, che hanno un misticismo lucido, han distinto due cose affatto differenti nello insieme delle opinioni che gli occidentali designano con quella sola parola. L'una di queste cose si chiama in arabo: "Wahhdatul-wugiûd" (l'unità dell'esistenza, cioè l'identità della creazione col Creatore). Qui è il panteismo quale domma definitivo, religioso e filosofico, e che noi non accettiamo. L'altra è il monoteismo vivente: l'unità nella pluralità e la pluralità nell'unità. [...] La differenza tra il monoteismo ch'è vivente e quello ch'è morto è la stessa ch'esiste tra la luce bianca del sole ed una macchia di gesso, bianca anch'essa; soltanto, l'una contiene tutti i colori e l'altra non ne ha alcuno» ${ }^{27}$.

Tuttavia, nei suoi articoli vi erano delle concezioni ed affermazioni che lasciano perplessi. Ad esempio, in un articolo della fine del $1907^{28}$, Agelii, avendo ritrovato dei manoscritti inediti del Sommo Maestro, volle comunicare la sua scoperta al pubblico, scrisse:

25 Anche se, a dire il vero, nel caso di Ibn 'Arabī le pubblicazioni in italiano, quanto a numero, sono del tutto irrilevanti; diremo dunque che la terminologia orientalista non si distanzia molto da quella da essa sviluppata per l'ambito teologico-filosofico islamico.

26 Il Convito, 1907, n. 3-4, p. 92.

27 Ibidem, pp. 101-2.

28 Il Convito, (n. ?), p. 195, non sono riuscito a reperire il numero della rivista, ma è probabile che sia uno degli ultimi mesi del 1907. 
«Ibn Arabi evoluzionista. Egli, nelle sue più importanti opere d'occultismo e di simbolismo, dice testualmente: «... Le cose create si combinano fra di loro; il loro abbassarsi consistè nel loro elevarsi, e l'elevarsi nell'abbassarsi. I minerali si trasformarono in piante, le piante in animali, gli animali nel genere umano e questo in minerali. Tutto fu moltiplicato con tutto, la forza si manifestò con l'azione...» Ecco l'espressione più semplice della teoria dell'evoluzione e della circolazione della materia. E fu emessa or sono sette secoli e solo ai nostri giorni, questo secolo di Saturno, fu sviluppata ampiamente da qualche sommo naturalista, Darwin fra i primi. Ora ò appunto parlando di Saturno, del suo ufficio e delle sue influenze, che il gran maestro ha detto quelle parole profonde che abbiamo riportato».

Queste affermazioni non devono tuttavia stupire più di tanto, data la formazione eclettica di Agelii. Sempre nel 1907, il pittore svedese curò il testo arabo e la traduzione italiana (parziale, poiché la pubblicazione de "Il Convito" s'interruppe in quell'anno) del trattato al-Sayr wa-l-sulūk ilā malik al-mulūk di Qāsim ibn Șalāḥ al-Dīn al-Ȟānī di Aleppo (1619-1697), tradotto da Agelii "Il progredire verso il Re dei Re" ${ }^{\prime 29}$. 